Proceedings

\title{
Cross-Linked Nanoparticle Membranes for Microelectromechanical Chemical Sensors and Pressure Sensors ${ }^{+}$
}

\author{
Hendrik Schlicke ${ }^{1,2, *}$, Hauke Hartmann ${ }^{2}$, Sophia Caroline Bittinger ${ }^{2}$, Matthias Rebber ${ }^{2}$, \\ Malte Behrens ${ }^{2}$ and Tobias Vossmeyer ${ }^{2}$ \\ 1 Fraunhofer Center for Applied Nanotechnology CAN, Grindelallee 117, 20146 Hamburg, Germany \\ 2 Institute of Physical Chemistry, University of Hamburg, Grindelallee 117, 20146 Hamburg, Germany; \\ hauke.hartmann@chemie.uni-hamburg.de (H.H.); sophia.bittinger@chemie.uni-hamburg.de (S.C.B.); \\ mrebber@physnet.uni-hamburg.de (M.R.); malte.behrens@uni-muenster.de (M.B.); \\ tobias.vossmeyer@chemie.uni-hamburg.de (T.V.) \\ * Correspondence: hendrik.schlicke@iap.fraunhofer.de; Tel.: +49-40-42838-8251 \\ † Presented at the Eurosensors 2018 Conference, Graz, Austria, 9-12 September 2018.
}

Published: 22 January 2019

\begin{abstract}
We present novel microelectromechanical chemical sensors for the detection of volatile organic compounds based on electrostatically actuated, nanometer-thin, freestanding membranes of organically cross-linked gold nanoparticles. The sensors employ quasi-static deflection amplitudes or resonance frequency shifts of the membranes as highly sensitive sensing signals and are capable of detecting analytes at concentrations down to the low ppm range. We show that the devices can further be utilized for analyte discrimination. Additionally, we demonstrate that freestanding GNP membranes can be used for the fabrication of highly sensitive resistive pressure sensors.
\end{abstract}

Keywords: gold; nanoparticles; nanocomposites; membranes; pressure sensors; chemical sensors; actuators; resonators; MEMS; NEMS

\section{Introduction}

Composite materials of ligand-stabilized or cross-linked gold nanoparticles (GNPs) attracted significant interest regarding sensing applications. The materials exhibit unique optical, mechanical, sorption and charge transport properties, which depend on the inorganic nanoparticles as well as the organic ligand matrix. Hence, their properties can be adjusted by incorporation of differently sized GNPs or selection of the ligands or cross-linking molecules. The electrical conductivity $\sigma$ of these composites is mediated by thermally activated tunneling processes [1] and can be well-described using Equation (1) [2],

$$
\sigma=\sigma_{0} e^{-\beta \delta} e^{-\frac{E_{\mathrm{A}}}{k T}}
$$

Here, $T$ is the temperature, $E_{\mathrm{A}}$ the charge transport activation energy, $\beta$ is the tunneling decay constant, and $\delta$ represents the interparticle spacing. When GNP composites experience strain, the latter is directly translated into changes of the interparticle distances, which-due to the highly sensitive tunneling based charge transport-result in strong conductivity changes. Hence, different studies addressed the application of substrate-supported GNP composites as highly sensitive strain gauges [3,4]. Recently, we demonstrated that the resistive strain sensitivity of freestanding GNP membranes can be exploited for the fabrication of highly sensitive pressure sensors with sensitivities of up to $\sim 10^{-4}$ mbar $^{-1}[5]$. 
Besides strain, sorption of analyte molecules significantly influences the GNP composites' conductivity. On the one hand, sorption results in swelling of the organic matrix surrounding the GNPs, which is translated in changes of $\delta$. On the other hand, sorption-induced variations of the organic matrix' dielectricity cause changes in the activation energy $E_{\text {A. }}$ Consequently, the applicability of GNP composites as chemiresistors (Figure 1a) with adjustable sensitivities and selectivities was extensively investigated [6-8].
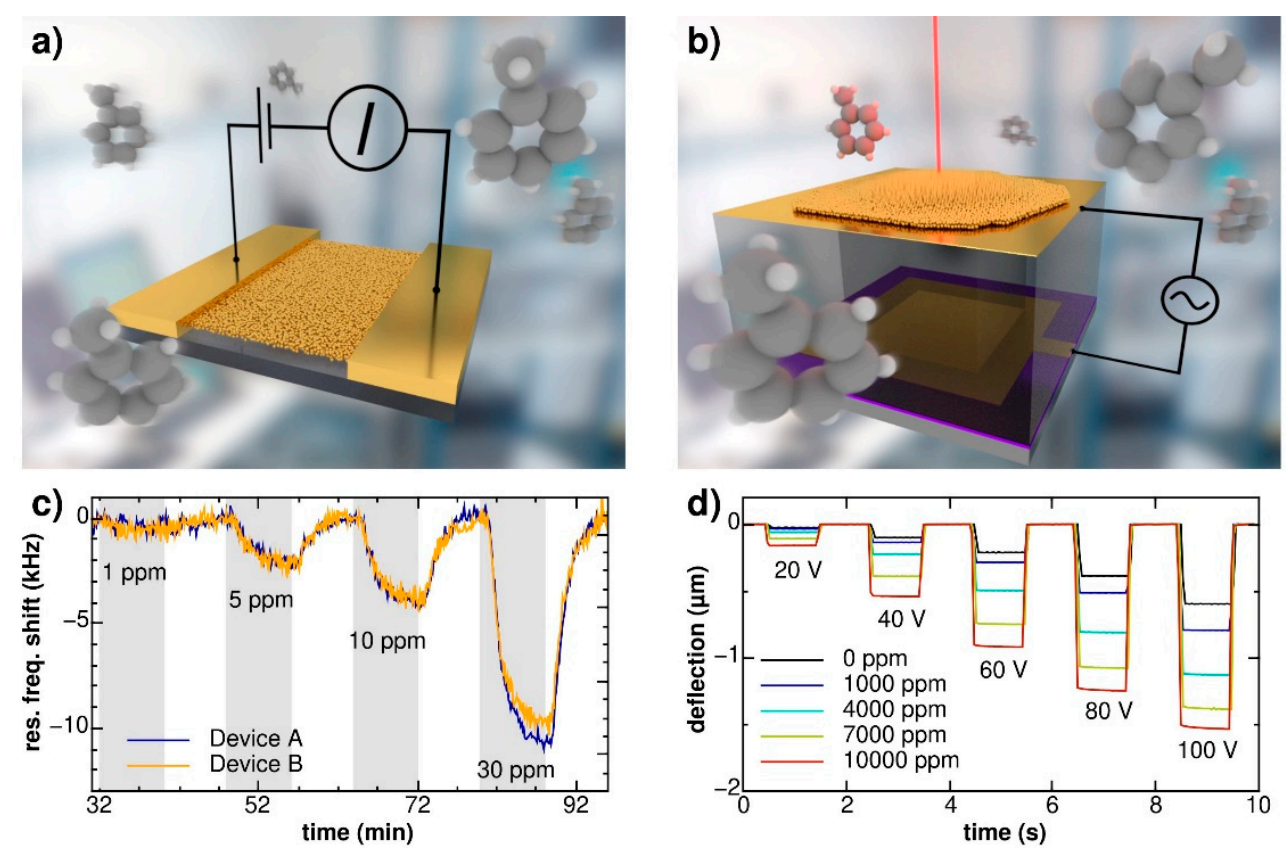

Figure 1. (a) Schematic depiction of a GNP composite film based chemiresistor; (b) Schematic depiction of a GNP membrane based microelectromechanical chemical sensor; (c) Frequency transients of GNP/6DT membrane based microelectromechanical chemical sensors under exposure to toluene vapors of different concentrations. The membrane edge lengths, thicknesses and fundamental resonance frequencies are $80 \mu \mathrm{m} / 60 \mu \mathrm{m}, 46 \mathrm{~nm} / 48 \mathrm{~nm}$ and $\sim 450 \mathrm{kHz} / 350 \mathrm{kHz}$ for devices A/B, respectively; (d) Quasi-static deflection transients of an electrostatically actuated GNP membrane based microelectromechanical chemical sensor under exposure to varying toluene concentrations (membrane edge length $100 \mu \mathrm{m}$, thickness $39 \mathrm{~nm}$ ) [13]. The experiments described in $\mathbf{c}$ and $\mathbf{d}$ were conducted under ambient pressure nitrogen atmosphere.

\section{Microelectromechanical Chemical Sensors}

Herein, we demonstrate the fabrication of microelectromechanical chemical sensors based on freestanding membranes of dithiol cross-linked GNPs. GNP composite films are first deposited onto sacrificial glass substrates and afterwards transferred onto microstructures featuring cavities. During transfer, freestanding membranes are formed, spanning the cavities (see Figure 1b). The microstructures are further equipped with two electrodes, one for establishing electric contact to the membranes and a counter electrode in proximity below the membranes. By applying a voltage between the electrode pairs, the membranes are charged against their counter electrodes and deflected towards the latter [9,10]. The deflection of the membranes is governed by an intrinsic membrane pre-stress, which is introduced during membrane transfer and forms the basis of the microelectromechanical sensing principle. We show that sorption of volatile organic compounds (VOCs) results in changes of this membrane pre-stress, leading to an altered dynamic or quasi-static membrane deflection behavior, which can be employed as sensing signal. 


\subsection{Dynamic Sensing Mode}

First, the tense membranes can be employed as drumhead resonators. The devices can be excited by AC signals (with an additional DC offset) applied to the electrode pairs. Upon matching a resonance frequency with the frequency-swept drive signal, the membranes show pronounced oscillations. The resonance frequencies of rectangular membranes can be described using Equation (2) [11]:

$$
f_{m, n}=\frac{1}{2} \sqrt{\frac{\sigma_{T}}{\rho}} \sqrt{\frac{m^{2}}{a_{x}^{2}}+\frac{n^{2}}{a_{y}^{2}}} .
$$

Here, $a_{x}$ and $a_{y}$ are the edge lengths of the membrane, $m$ and $n$ are integers corresponding to the vibrational mode of the membrane, and $\sigma_{\mathrm{T}}$ as well as $\rho$ denote the membrane pre-stress and mass density, respectively. Figure 1c depicts the fundamental resonance frequency transients of two 1,6hexanedithiol (6DT) cross-linked freestanding square GNP membranes repeatedly exposed to toluene vapor of different concentrations. Toluene concentrations as low as $5 \mathrm{ppm}$ could be easily detected using the sensors. Along similar experiments conducted at a lowered total pressure $(\sim 20$ mbar) involving similar toluene partial pressures $(\sim 2-20 \mathrm{~Pa})$ quartz-crystal microbalance (QCM) measurements were performed to observe the mass uptake of the GNP membranes. Hereby, it was shown that changes of the GNP membrane density play a minor role and the sensor response is mainly governed by changes in $\sigma \mathrm{T}$ for the investigated system [12].

\subsection{Quasi-Static Sensing Mode}

Besides using the membranes' resonance frequencies as sensing signals, also quasi-static membrane deflections in response to the application of DC voltage signals between the electrode pair can be employed as a sensing signal [13]. Figure 1d shows deflection transients of a 6DT cross-linked GNP membrane to periodically applied voltages, in pure nitrogen atmosphere, as well as under exposure to toluene vapors of different concentrations. Assuming small deflections and a constant, homogeneous electric field, the membranes' central point deflections $h$ under applied voltages $V$ are related to the membranes' pre-stress as approximated in relation 3 [9]:

$$
V^{2} \propto \sigma_{T} h \text {. }
$$

\section{Multivariate Sensing}

By combination of the different transduction methods, devices enabling multivariate sensing using only a single GNP membrane can be realized. Here, different selectivities arising from the different transduction principles enable analyte discrimination. Figure 2a exemplarily shows the frequency response of a GNP membrane resonator as well as the chemiresistive response of a GNP film section, fabricated from another section of the GNP film, under exposure to toluene, 4methylpentan-2-one (4m2p), 1-propanol and water $\left(\mathrm{H}_{2} \mathrm{O}\right)$. The plots show different selectivities that could be used as input parameters for analyte recognition models. Further, Figure $2 b$ shows the normalized response transients of a GNP membrane resonator under exposure to vapors of different VOCs. Clearly different response kinetics, presumably related to analyte diffusion, were observed for the analytes, enabling the use of the device's response time/shape for analyte recognition. 

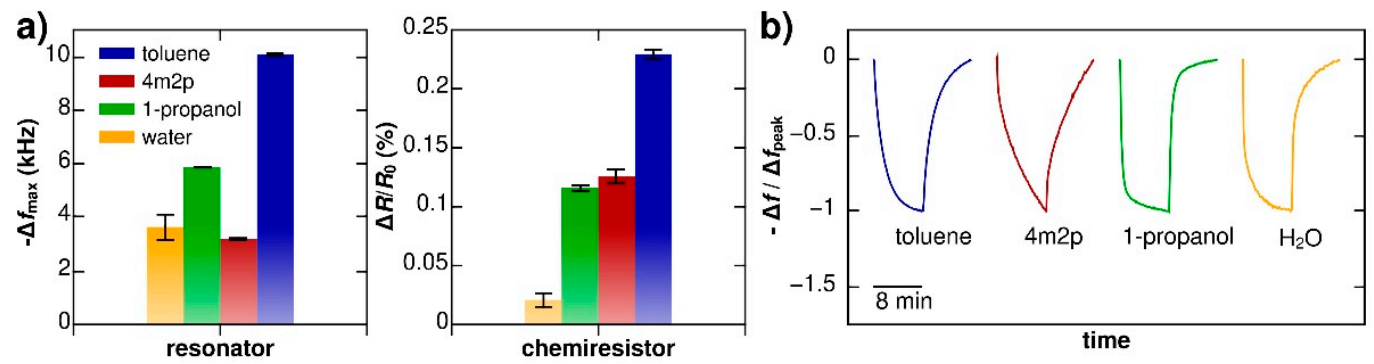

Figure 2. (a) Frequency shift responses of a GNP membrane resonator to exposures to different VOCs (8 min exposure, 10,000 ppm, 20 mbar total pressure, $\mathrm{N}_{2}$ atmosphere) and resistive responses of another section of the same GNP composite film to the exposures; (b) Response kinetics of the GNP membrane resonator under transient exposure to the different VOCs [12].

\section{Conclusions}

In this contribution we reported novel microelectromechanical chemical sensors based on electrostatically actuated membranes of organically cross-linked GNPs. Two transduction mechanisms based on the excitation of quasi-static deflections or resonant vibrations of the membranes were employed to detect VOCs. Further, we showed that the combination of these transduction principles with an additional chemiresistive readout of GNP composite membranes enables multivariate sensing and analyte recognition. Here, differing selectivities due to different transduction mechanisms and/or analyte-dependent response kinetics of the sensors yield patterns that can be assigned to certain analytes.

Funding: T.V. acknowledges financial support by the DFG, grant number VO698/3-1.

Author Contributions: H.S. developed the different sensing devices and contributed to major extent to the device design, the experimental design and execution of characterization experiments as well as data interperation. H.H., S.C.B., M.R., and M.B. gave important contributions to the device fabrication and conducted characterization experiments. T.V. was involved as project leader contributing to the experimental design and data interpretation and by setting the scientific objectives. The manuscript was written by H.S. and finalized together with T.V. taking into account the input of all coauthors.

Conflicts of Interest: The authors declare no conflict of interest.

\section{References}

1. Brust, M.; Schiffrin, D.J.; Bethell, D.; Kiely, C.J. Novel gold-dithiol nano-networks with non-metallic electronic properties. Adv. Mater. 1995, 7, 795-797.

2. Terrill, R.H.; Postlethwaite, T.A.; Chen, C.-H.; Poon, C.-D.; Terzis, A.; Chen, A.; Hutchison, J.E.; Clark, M.R.; Wignall, G.; Londono, J.D.; et al. Monolayers in Three Dimensions: NMR, SAXS, Thermal, and Electron Hopping Studies of Alkanethiol Stabilized Gold Clusters. J. Am. Chem. Soc. 1995, 117, 12537-12548.

3. Herrmann, J.; Müller, K.-H.; Reda, T.; Baxter, G.R.; Raguse, B.; de Groot, G.J.J.B.; Chai, R.; Roberts, M.; Wieczorek, L. Nanoparticle films as sensitive strain gauges. Appl. Phys. Lett. 2007, 91, 183105.

4. Vossmeyer, T.; Stolte, C.; Ijeh, M.; Kornowski, A.; Weller, H. Networked Gold-Nanoparticle Coatings on Polyethylene: Charge Transport and Strain Sensitivity. Adv. Funct. Mater. 2008, 189, 1611-1616.

5. Schlicke, H.; Rebber, M.; Kunze, S.; Vossmeyer, T. Resistive pressure sensors based on freestanding membranes of gold nanoparticles. Nanoscale 2016, 8, 183-186.

6. Wohltjen, H.; Snow, A.W. Colloidal Metal-Insulator-Metal Ensemble Chemiresistor Sensor. Anal. Chem. 1998, 70, 2856-2859.

7. Olichwer, N.; Meyer, A.; Yesilmen, M.; Vossmeyer, T. Gold nanoparticle superlattices: Correlating chemiresistive responses with analyte sorption and swelling. J. Mater. Chem. C 2016, 4, 8214-8225.

8. Ibanez, F.J.; Zamborini, F.P. Chemiresistive Sensing with Chemically Modified Metal and Alloy Nanoparticles. Small 2012, 8, 174-202.

9. Schlicke, H.; Battista, D.; Kunze, S.; Schröter, C.J.; Eich, M.; Vossmeyer, T. Freestanding Membranes of Cross-Linked Gold Nanoparticles: Novel Functional Materials for Electrostatic Actuators. ACS Appl. Mater. Interfaces 2015, 7, 15123-15128. 
10. Schlicke, H.; Schröter, C.J.; Vossmeyer, T. Electrostatically driven drumhead resonators based on freestanding membranes of cross-linked gold nanoparticles. Nanoscale 2016, 8, 15880-15887.

11. Zhang, X.; Waitz, R.; Yang, F.; Lutz, C.; Angelova, P.; Gölzhäuser, A.; Scheer, E. Vibrational modes of ultrathin carbon nanomembrane mechanical resonators. Appl. Phys. Lett. 2015, 106, 063107.

12. Schlicke, H.; Behrens, M.; Schröter, C.J.; Dahl, G.T.; Hartmann, H.; Vossmeyer, T. Cross-Linked GoldNanoparticle Membrane Resonators as Microelectromechanical Vapor Sensors. ACS Sens. 2017, 2, 540-546.

13. Schlicke, H.; Bittinger, S.C.; Behrens, M.; Yesilmen, M.; Hartmann, H.; Schröter, C.J.; Dahl, G.T.; Vossmeyer, T. Electrostatically Actuated Membranes of Cross-Linked Gold Nanoparticles: Novel Concepts for Electromechanical Gas Sensors. Proceedings 2017, 1, 301.

(C) 2018 by the authors. Licensee MDPI, Basel, Switzerland. This article is an open access article distributed under the terms and conditions of the Creative Commons Attribution (CC BY) license (http://creativecommons.org/licenses/by/4.0/). 